\title{
Successful Treatment of Chronic Monteggia Fracture-dislocations in Children-A New Reference
}

\section{Ping Xu}

Children's Hospital of Fudan University

\section{Zhiqiang Zhang}

Children's Hospital of Fudan University

\section{Bo Ning}

Children's Hospital of Fudan University

Dahui wang ( $\nabla$ wangdahui@fudan.edu.cn )

Children's Hospital of Fudan University

\section{Research Article}

Keywords: Missed Monteggia fracture, children, radial head dislocation

Posted Date: July 30th, 2021

DOI: https://doi.org/10.21203/rs.3.rs-732196/v1

License: (c) (1) This work is licensed under a Creative Commons Attribution 4.0 International License. Read Full License 


\section{Abstract}

\section{Background}

Great difficulty and more failures were the descriptions of a chronic Monteggia fracture-dislocation. The treatment of chronic Monteggia lesion remains controversial and challenging for surgeons. This study aims to introduce our experience of a new reference in the treatment of chronic Monteggia fracturedislocation in children and evaluate outcomes from clinical and radiographic findings.

\section{Methods}

We retrospectively reviewed 18 children who underwent surgical treatment because of chronic Monteggia lesion. Electronic medical records of clinical data, radiographic parameters, and operative details, were reviewed for study analysis. Parameters were compared at the time of pre-operation and the last followup. The relationship of lengthening and angulation of ulnar was calculated.

\section{Results}

Mean interval time was $11.1 \pm 15.7$ months and follow-up time was $34.6 \pm 23.7$ months in this study. A congruent radiocapitellar reduction was observed in 15 (83.3\%) patients, while $2(11.1 \%)$ patients developed subluxation, and 1 (5.6\%) patient had redislocation. The mean posterior bending angle was $12.88^{\circ}$ (range, 3 to $25^{\circ}$ ), and the mean amount of elongation of the ulnar was $8.78 \mathrm{~mm}$ (range, 3.6 to 17.5 $\mathrm{mm}$ ). The lengthening was significantly proportional to the magnitude of angulation of ulnar in good outcome patients $(r=0.637, p=0.009)$, and the index was larger than the failed ones. Postoperatively, the Kim scores were obviously improved, from $59.17 \pm 18.17$ to $90 \pm 6.64$.

\section{Conclusions}

We highlight the ulnar osteotomy as the essential procedure during the reconstruction surgeries. Enough elongation and balanced angulation of the osteotomy is warranted to keep satisfactory outcomes. The ulnar should be lengthened to more than normal proportional ulnar length to stable the radial head reduction. Iliac crest autograft is recommended to avoid nonunion of ulnar osteotomy after enough lengthening.

Level of evidence

Level IV; Case Series; Treatment Study

\section{Background}

Monteggia fracture-dislocation, first described by Giovanni Monteggia in 1814, is a fracture of the ulna associated with dislocation of the radiocapitellar joint and disruption of the proximal radioulnar joint [1]. When the Monteggia lesion is identified in the acute situation, the outcomes are always achieved well. 
However, many of these injuries in children are chronic at initial presentation, for adult orthopedics evaluation, inadequate radiographs, subtle greenstick fractures of the ulna, bowing of ulna, and the complexity of evaluating the pediatric elbow its multiple ossification centers[2]. The neglected lesion's common presentation is of bony prominence, limited range of motion (ROM), valgus deformity of the elbow, with or without elbow pain, limited supination and pronation of the forearm, and neurological problems[3-6].

The treatment of chronic Monteggia lesion remains controversial and challenging for surgeons. It is recommended that once the diagnosis has been made, the open reduction and ulnar osteotomy with or without reconstruction of the annular ligament should be performed since the rate of radius and ulnar is changed, the shape of radial head hypertrophies and the annular ligament and associated anterior capsular structures becoming a block to anatomic reduction[6-9], Nevertheless, there remains no consensus gold-standard treatment at this time. Previously, we found that the proportional ulnar length (PUL), defined as the length of the ulna/length of the radius, consistently averaged approximately 1.1 on healthy children[10]. Therefore, we decided to compare the pre-op PUL to the final follow-up PUL, trying to reveal the proportional change of chronic Monteggia lesion.

Since 2008, 18 chronic Monteggia lesion patients in our center were treated by open reduction and ulnar osteotomy with or without annular ligament reconstruction. This study aims to report our experience of the new reference and evaluate it from clinical and radiographic findings.

\section{Methods}

After the institution's Ethics Committee approval, a retrospective review was performed in all patients diagnosed with Chronic Monteggia lesion from January 2008 to May 2019 who underwent surgery reconstruction. Bone tumors, secondary radial head dislocation, or congenital diseases were excluded.

The mean age at the time of surgery, and the mean time interval from injury to surgery was calculated. The clinical data of Bado type[11], complaints before operation, pre-and post-operational Kim score [12], complications and satisfaction of parents (Table 1) were collected. Based on the clinical observation, the cosmetic and ROM problems are the primary concerns, thus, we described three terms of outcomes by parents.

Table 1

Satisfaction of parents

\begin{tabular}{|lll|}
\hline Satisfaction & Description & No. of patients \\
\hline Satisfied & The complains are solved and nothing unusual in daily life. & 17 \\
Good & Some activities require help, but no pain, no cosmetic problems. & 1 \\
Poor & Pain, cosmetic problems and/or daily activities are restricted. & 0 \\
\hline
\end{tabular}


The radiological parameters of maximum interosseous distance (MID) from anteroposterior view, pre-and post-operative PUL from a lateral view, lengthening of ulnar (the biggest distance between the two sites of osteotomy after the ulnar lengthening), and the bending angle of ulnar from a lateral view and the radiological evaluation results on the last follow-up was summarized.

The surgeries were performed under general anesthesia. A 3 to $4 \mathrm{~cm}$ incision was made to access necessary structures. The radial nerve was identified, depressed, and protected. Then, the elbow joint was opened, the radial head was exposed, and the state of the cartilage of the radial head and annular ligament were assessed. Reduce the ligament if it exists and complete; excise fibrous that impedes reduction of the radial head. Another 4 to $6 \mathrm{~cm}$ incision was achieved to have a proximal ulnar osteotomy. Two factors determined the bending angle and the elongation distance of the ulna. First, we decided to lengthen the ulna to restore the PUL to more than the normal value of 1.1. Second, confirm the position of the radial head by means of direct observation. (flexion, extension, supination, pronation). Inserting an allograft or autogenous cancellous iliac crest bone graft at the ulnar osteotomy site. Then 5- or 6-holes plate was placed at the lateral (or medial) side instead of backside of ulnar (Fig. 1). The limb was placed into a long-arm cast, with the elbow in 90 to 100 degrees of flexion and the forearm in supination. Cast immobilization was maintained for 6 weeks, then, the exercises were initiated. Six months later, after the ulnar union, removed the implant.

The Kolmogorov-Smirnov test analyzed the continuous variable to assess for normality. Comparisons of two groups in terms of PUL, MID, and Kim scores were performed by paired-t-test. We tested the correlation between the length of ulnar and the bending angle by the Spearman nonparametric correlation test. Two single doctors independently measured and collected all data. Statistical analysis was performed using SPSS 19.0 (IBM, America). A p-value $<0.05$ was considered significant for all statistical tests.

\section{Results}

Eighteen patients, 15 boys (83.3\%) and 3 girls, were included. There were 11 right-sided injuries (61.1\%). Mean age at initial treatment was $6.78 \pm 2.67$ years old, ranging from 4 to 13 years old. Mean interval time was $11.1 \pm 15.7$ months, ranging from 1 to 48 months. Mean follow-up time was $34.6 \pm 23.7$ months (range, 7-84 months). According to Bado classification, 16 patients were classified as type I, and 2 patients were type III. The most prominent complains were cosmetic problems and limited ROM. (Table 2) 
Table 2

Demographic details of patients

\begin{tabular}{|ll|}
\hline Demographic Data & \\
\hline Sex (No. of patients) & 15 \\
\hline Male & 3 \\
\hline Female & \\
\hline Side (No. of patients) & 7 \\
\hline Left & 11 \\
\hline Right & 10 \\
Complains & 15 \\
bony prominence & 5 \\
limited range of motion & 3 \\
pain & 2 \\
valgus deformity & 34.58 (7-84) \\
\hline neurological problems & 3 \\
\hline Follow up time* (mon) & 1 \\
\hline Complications & \\
\hline Nonunion & \\
Redislocation & *The values are given as the mean, with the range in parentheses. \\
\hline
\end{tabular}

The details of the reconstructions were showed in Table 3. The annular ligaments were repaired and repositioned in $1(5.6 \%)$ and 7 (38.9\%) patients, respectively, while $10(55.6 \%)$ patients had excised scar tissues without any reconstruction. Notably, three patients had allograft insertion after ulnar lengthening (mean $1.5 \mathrm{~cm}$ ) and angulation (mean $22.5^{\circ}$ ); after more than 6-months of follow-up, nonunion was observed (Fig. 2). Then, reoperation and autogenous cancellous iliac crest bone graft insertion was performed. Finally, they all had a good outcome. 2 patients had the symptom of radial nerve issues and recovered several months later after surgery. A congruent radiocapitellar reduction was observed in 15 (83.3\%) patients, while $2(11.1 \%)$ patients developed subluxation, and $1(5.6 \%)$ patient had redislocation. The mean posterior bending angle was $12.88^{\circ}$ (range, 3 to $25^{\circ}$ ), and the mean amount of elongation of the ulna was $8.78 \mathrm{~mm}$ (range, 3.6 to $17.5 \mathrm{~mm}$ ) (Table 4). The relationship of good outcome patients and others were seen in the Fig. 3. The lengthening was directly proportional to the magnitude of angulation of ulnar in good outcome patients $(r=0.637, p=0.009)$, and the index was larger than the failed group. The relationship between interval time and lengthening and angulation was shown in the Fig. 4. No 
apparent correspondent relationship was noticed. Radiological changes were recorded. The PUL and MID were significantly increased respectively from pre-operation to the latest follow-up. Postoperatively, the Kim scores were significantly improved, from $59.17 \pm 18.17$ to $90 \pm 6.64$. (Table 5)

Table 3

Surgical details of patients

\begin{tabular}{|lc|}
\hline Surgical Data & $6.78(4-13)$ \\
\hline Age* $(\mathrm{y})$ & $11.1(1-48)$ \\
\hline Interval time* (mon) & \\
\hline Annular ligament & 1 \\
\hline Repair & 10 \\
\hline Excised scar tissue & 7 \\
Reposition & \\
\hline Ulnar osteotomy & 0 \\
\hline CORA & 18 \\
Proximal site & 12 \\
\hline Bone graft & 4 \\
lliac & 3 \\
Allograft & 3 \\
Nonunion & 1 \\
\hline Complications & 2 \\
Bone nonunion & \\
Redislocation & \\
Subluxation & \\
*The values are given as the mean, with the range in parentheses. & \\
\hline
\end{tabular}


Table 4

Radiological details of petients

\begin{tabular}{|ll|}
\hline Radiographic Data \\
\hline Bado classification \\
\hline I III & 16 \\
\hline Final reduction status & 2 \\
\hline Good & 15 \\
Fair & 2 \\
Poor & 1 \\
\hline Arthrosis & 0 \\
\hline Lengthening* $(m m)$ & $8.78(3.6-17.5)$ \\
\hline Osteotomy angle* ${ }^{\circ}$ ) & $12.88(3-25)$ \\
\hline$*$ The values are given as the mean, with the range in parentheses. \\
\hline
\end{tabular}

Table 5

Radiological and clinical changes pre- and post-surgeries

\begin{tabular}{|llllll|}
\hline \multicolumn{5}{|l|}{ Radiological and clinical changes } \\
\hline & Preop.* & Latest Follow-up* & Difference & $95 \% \mathrm{Cl}$ & $P$ value \\
\hline PUL & $1.06 \pm 0.03$ & $1.13 \pm 0.11$ & -0.07 & -0.03 to -0.00 & 0.047 \\
\hline MID & $1.11 \pm 0.26$ & $1.28 \pm 0.27$ & -0.17 & -0.32 to -0.03 & 0.021 \\
\hline Kim scores & $59.17 \pm 18.17$ & $90 \pm 6.64$ & -35.83 & -45.86 to -25.81 & 0.000 \\
\hline \multicolumn{7}{|l}{ *The values are given as the mean and the standard deviation. } \\
\hline
\end{tabular}

\section{Discussion}

Great difficulty and more failures were the descriptions of a chronic Monteggia fracture-dislocation. A great advance has been witnessed recently, focusing on exploring the better surgery reduction outcome; however, the consensus has never been made about a perfect protocol procedure to achieve satisfactory results. Our surgical procedure is based on the hypothesis that the ulnar osteotomy plays the most crucial role in achieving stable radial head reduction; hence, we calculated a new reference of PUL for the first time using in Monteggia disease, focusing on providing valuable information for the clinical use. 
The ligament reconstruction technique was changed a lot and controversial. Forearm fascia, the remnant annular ligament, biceps or triceps tendon, and palmaris longus tendon graft were reported for the annular ligament reconstruction[13-15], for which was recommended for the importance in stabilizing the radioulnar joint and the forearm rotation $[16,17]$. However, Rahbek and cols[18] had reconstructed the ligament in 10 patients and compared with six patients not did it, having no difference in the final result. Soni et al.[19] also reported a good outcome without ligament reconstruction in any six patients. Delmont et al. also performed a multicenter study comparing the ulnar osteotomy with annular ligament reconstruction or without it, no significant difference in clinical, functional outcomes between groups was found. In our research, open reduction of the radial head with remnant annular ligament repositioning or without ligament repairing and reconstruction was performed to obtain the reduction. However, the maintenance of this reduction should be achieved by optical ulnar osteotomy and enough lengthening.

Several issues should be addressed about ulnar osteotomy. The first one is the position of the osteotomy. Center of rotation angulation (CORA) and proximal part of ulnar metaphysis were the most mentioned position for osteotomy. However, the nonunion or delayed union problem was more prominent at the CORA site[20, 21]. Ulnar malunion was also blamed for the difficult of the radial head reduction[20]. Moreover, a proximal osteotomy was recommended for having an unnoticeable posterior curvature and avoiding forearm rotation restriction because the whole interosseous membrane (IOM) remains constant[18]. The second issue is quantitative of ulnar elongation and posterior bending angle. From our data, the more lengthening, the more angulation was needed. Three patients failed the reduction having an insufficient lengthening or angulation. Then, we ameliorated the technique based on the value of PUL, we concluded that the lengthening of the ulnar to more than the normal PUL was warranted. An open wedge angle proportional to the elongation was also needed, which allows for a stable reduction of the radial head in flexion, extension, pronation, and supination. In this way, the outcomes turn out well. This kind of osteotomy not only leaves room for the radial head reduction but also tensions the IOM against forces that would lead to recurrent radial head dislocation[4, 22].

After osteotomy, different internal or external fixation was reported to fix the ulnar. David et al. [14] reported the K-wire to stabilize the osteotomy and had no complications in 6 patients. Rahbek et al.[18] also stated that rigid fixation or pin with less stability could stabilize the osteotomy. However, this construct only provides relative stability, which may be inadequate for the older patients, the proximal osteotomy site, and maintaining the angular correction. Recently, external fixation has been reported to be a safe and effective methods to treat this injury. Bor, Yuan, and Lu [23-25] all reported their experience using external fixation to capture the ulna osteotomy's optimal position to achieve the best possible reduction of the radial head. However, wearing huge external fixation for several months might leave psychological problems. Pin tract infection, neurovascular problems, malunion, and the delayed union should also be addressed for this construction. Nowadays, rigid fixation with plate is more accepted, allowing for a post-operative ROM, minimizing contracture[26]. We modified the technique by putting plate into the ulnar's lateral or medial side instead of the backside. First, it could diminish the stress applied to the plate without plate bending, which reduces the risk of plate breakage. Second, it is more aesthetic and comfortable for patients because the palpable pain or bump will vanish. 
The IOM has been reported to play an important role in the longitudinal and transverse stability of the forearm[27]. The recommended osteotomy on the proximal side also maintains the IOM's stouter distal fibers, better translating ulnar correction to the intact radius [26]. Soubeyrand et al[27] describe this structure as the middle radioulnar joint, which is a fibrous joint, allowing pronosupination and ensuring forearm stability. From our research, the obvious difference of MID between pre-operation with the final follow-up of these successful cases also explained the functional effect of IOM. As for the challenging patients whose radial head unstable after the scar tissue is dissected and the elongation and angulation of ulnar are finished, we might increase supination slightly, and then, the radial head was reduced. This fact highlights the crucial role played by the IOM in forearm stability.

The present study had limitations. First, this is a retrospective study to evaluate PUL's value in the treatment of chronic Monteggia lesion, however, this is the first to bring up this reference; more randomized controlled trials or prospective studies are required for further validation. Second, all patients need longer follow-up to confirm if there are any delayed redislocation.

In conclusion, we emphasize the importance of the ulnar osteotomy to obtain and maintain reduction of the radiocapitellar joint in chronic Monteggia lesions by addressing both length and angular. Enough elongation to at least normal PUL and balanced angulation of the osteotomy is warranted to keep satisfactory outcomes. It is more aesthetic and safer to put the plate into the lateral or medial side of the ulnar. The functional effect of IOM cannot be neglected during the surgeries. Iliac crest autograft is recommended to avoid nonunion of ulnar osteotomy after lengthening.

\section{Declarations}

\section{Ethics approval and consent to participate}

Our study was approved by The Ethics Committee approval of Children's hospital of fudan university, shanghai. China

\section{Consent for publication}

All volunteers agreed to participate in research and consent for publication.

\section{Availability of data and materials}

The datasets used and/or analyzed during the current study are available from the corresponding author on reasonable request.

\section{Competing interests}

The authors declare that they have no competing interests.

\section{Funding}


The project is sponsored by Shanghai Shenkang Clinical Research Cultivation Project (2019). NO. SHDC12019X28

\section{Authors' contributions}

Zhiqiang Zhang and Ping Xu made substantial contributions to the conception, reviewed the case, collected the data, carried out the initial analyses, and drafted the initial manuscript. Bo Ning performed the surgeries. Dahui Wang coordinated and supervised data collection, and critically reviewed the revised the manuscript. All authors read and approved the final manuscript.

\section{Acknowledgements}

Not applicable

\section{Conflict of Interest of Statement}

Each author certifies that he or she has no commercial associations that might pose a conflict of interest in connection with the submitted article.

\section{References}

1. Ring D, Waters PM. Operative fixation of Monteggia fractures in children. The Journal of bone joint surgery British volume. 1996;78(5):734-9.

2. Fowles JV, Sliman N, Kassab MT. The Monteggia lesion in children. Fracture of the ulna and dislocation of the radial head. The Journal of bone joint surgery American volume. 1983;65(9):1276-82.

3. Rodgers WB, Waters PM, Hall JE. Chronic Monteggia lesions in children. Complications and results of reconstruction. The Journal of bone joint surgery American volume. 1996;78(9):1322-9.

4. Inoue G, Shionoya K: Corrective ulnar osteotomy for malunited anterior Monteggia lesions in children. 12 patients followed for 1-12 years. Acta orthopaedica Scandinavica 1998, 69(1):73-76.

5. Eamsobhana P, Chalayon O, Kaewpornsawan K, Ariyawatkul T: Missed Monteggia fracture dislocations treated by open reduction of the radial head. The bone \& joint journal $2018,100-$ b(8):1117-1124.

6. Hubbard J, Chauhan A, Fitzgerald R, Abrams R, Mubarak S, Sangimino M. Missed Pediatric Monteggia Fractures. JBJS reviews. 2018;6(6):e2.

7. Kim HT, Conjares JN, Suh JT, Yoo Cl. Chronic radial head dislocation in children, Part 1: pathologic changes preventing stable reduction and surgical correction. J Pediatr Orthop. 2002;22(5):583-90.

8. Wilkins KE. Changes in the management of monteggia fractures. J Pediatr Orthop. 2002;22(4):54854.

9. Bhaskar A. Missed Monteggia fracture in children: Is annular ligament reconstruction always required? Indian journal of orthopaedics. 2009;43(4):389-95. 
10. Huang P, Zhu L, Ning B. Forearm Deformity and Radial Head Dislocation in Pediatric Patients with Hereditary Multiple Exostoses: A Prospective Study Using Proportional Ulnar Length as a Scale to Lengthen the Shortened Ulna. The Journal of bone joint surgery American volume. 2020;102(12):1066-74.

11. Bado JL. The Monteggia lesion. Clin Orthop Relat Res. 1967;50:71-86.

12. Kim HT, Park BG, Suh JT, Yoo Cl. Chronic radial head dislocation in children, Part 2: results of open treatment and factors affecting final outcome. J Pediatr Orthop. 2002;22(5):591-7.

13. Lloyd-Roberts GC, Bucknill TM: Anterior dislocation of the radial head in children: aetiology, natural history and management. The Journal of bone and joint surgery British volume 1977, 59-b(4):402407.

14. David-West KS, Wilson NI, Sherlock DA, Bennet GC. Missed Monteggia injuries. Injury. 2005;36(10):1206-9.

15. Gyr BM, Stevens PM, Smith JT. Chronic Monteggia fractures in children: outcome after treatment with the Bell-Tawse procedure. Journal of pediatric orthopedics Part B. 2004;13(6):402-6.

16. Nakamura K, Hirachi K, Uchiyama S, Takahara M, Minami A, Imaeda T, Kato H. Long-term clinical and radiographic outcomes after open reduction for missed Monteggia fracture-dislocations in children. The Journal of bone joint surgery American volume. 2009;91(6):1394-404.

17. Seel MJ, Peterson HA. Management of chronic posttraumatic radial head dislocation in children. $J$ Pediatr Orthop. 1999;19(3):306-12.

18. Rahbek O, Deutch SR, Kold S, Søjbjerg JO, Møller-Madsen B. Long-term outcome after ulnar osteotomy for missed Monteggia fracture dislocation in children. J Child Orthop. 2011;5(6):449-57.

19. Soni JF, Valenza WR, Matsunaga CU, Costa ACP, Faria FF. CHRONIC MONTEGGIA FRACTUREDISLOCATION IN CHILDREN SURGICAL STRATEGY AND RESULTS. Acta ortopedica brasileira. 2019;27(5):244-7.

20. Lädermann A, Ceroni D, Lefèvre Y, De Rosa V, De Coulon G, Kaelin A. Surgical treatment of missed Monteggia lesions in children. J Child Orthop. 2007;1(4):237-42.

21. Wang MN, Chang WN. Chronic posttraumatic anterior dislocation of the radial head in children: thirteen cases treated by open reduction, ulnar osteotomy, and annular ligament reconstruction through a Boyd incision. J Orthop Trauma. 2006;20(1):1-5.

22. Song KS, Ramnani K, Bae KC, Cho CH, Lee KJ, Son ES. Indirect reduction of the radial head in children with chronic Monteggia lesions. J Orthop Trauma. 2012;26(10):597-601.

23. Lu X, Kun Wang Y, Zhang J, Zhu Z, Guo Y, Lu M. Management of missed Monteggia fractures with ulnar osteotomy, open reduction, and dual-socket external fixation. J Pediatr Orthop. 2013;33(4):398-402.

24. Yuan Z, Xu HW, Liu YZ, Li YQ, Li JC, Canavese F. The use of external fixation for the management of acute and chronic Monteggia fractures in children. J Child Orthop. 2019;13(6):551-9. 
25. Bor N, Rubin G, Rozen N, Herzenberg JE. Chronic anterior monteggia lesions in children: report of 4 cases treated with closed reduction by ulnar osteotomy and external fixation. J Pediatr Orthop. 2015;35(1):7-10.

26. Horii E, Nakamura R, Koh S, Inagaki H, Yajima H, Nakao E. Surgical treatment for chronic radial head dislocation. The Journal of bone joint surgery American volume. 2002;84(7):1183-8.

27. Soubeyrand M, Wassermann V, Hirsch C, Oberlin C, Gagey O, Dumontier C. The middle radioulnar joint and triarticular forearm complex. The Journal of hand surgery European volume. 2011;36(6):447-54.

\section{Figures}

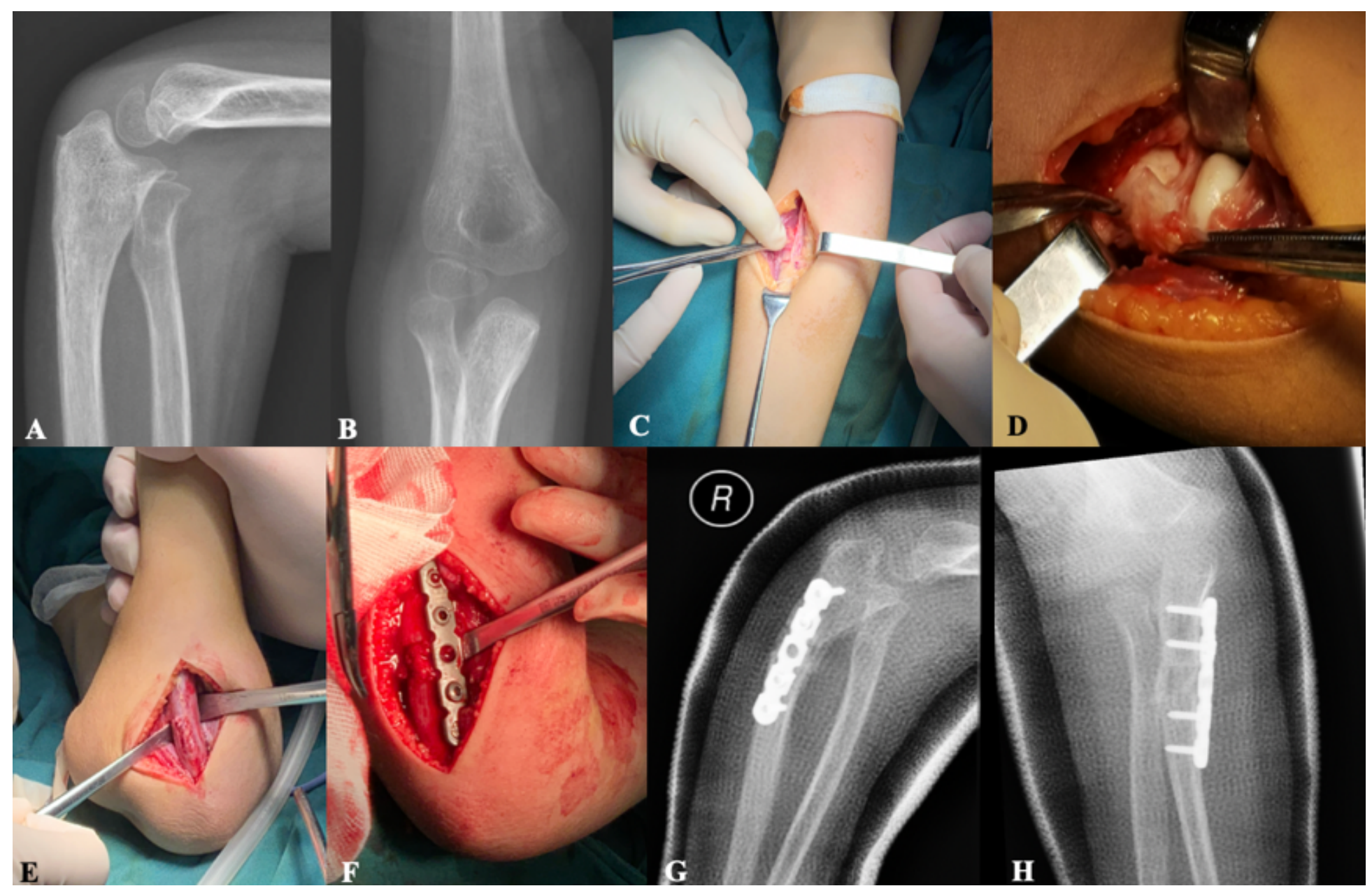

Figure 1

Demonstration of our surgical procedure: $\mathrm{A}$ and $\mathrm{B}$ : anteroposterior and lateral view of the elbow before the surgery; $C$ : $A 3$ to $5 \mathrm{~cm}$ incision was made to access necessary structures. The radial nerve was identified, depressed, and protected; D: The state of the cartilage of the radial head and annular ligament were assessed, reduce the ligament or excise the scar tissues; E: Another 4 to $6 \mathrm{~cm}$ incision was achieved to have a proximal ulnar osteotomy; F: Insert an autogenous cancellous iliac crest bone graft at the ulnar 
osteotomy site, then 5- or 6-holes plate was placed at the medial (or lateral) side of ulnar; $\mathrm{G}$ and $\mathrm{H}$ : lateral and anteroposterior view of the elbow in the surgery.

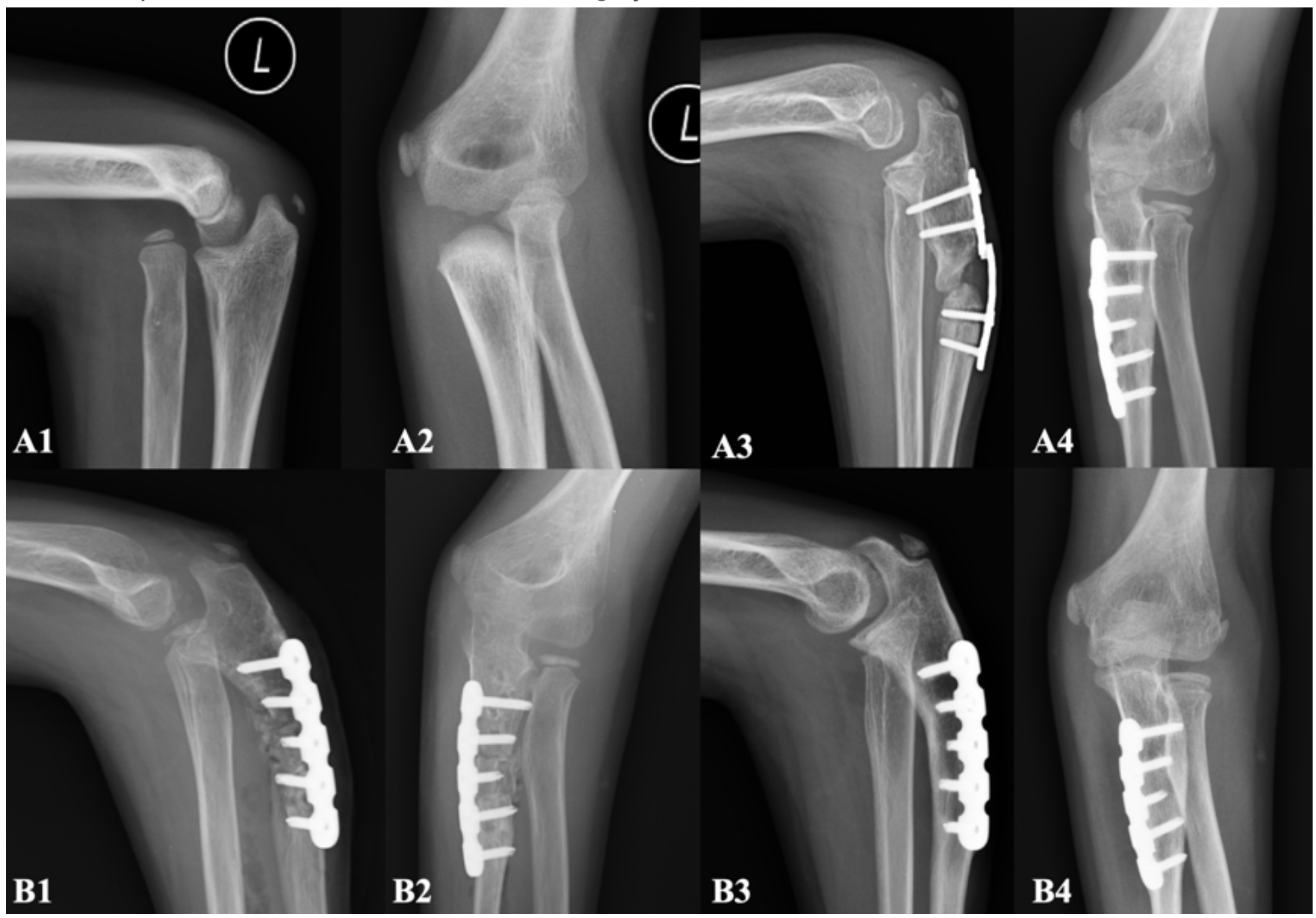

Figure 2

The radiological data of one of the nonunion patients. A 15-year-old boy with a left chronic Monteggia fracture-dislocation (Bado type III) for 4 years underwent traditional open reduction procedure. A1-2, the radial head was dislocated in an anterior position; A3-4, 6 months later, nonunion was observed; B1-B2, reoperation and autogenous cancellous iliac crest bone graft insertion was performed; B3-4, one year after the second surgery, the nonunion healed well. 


\section{Final reduction status}

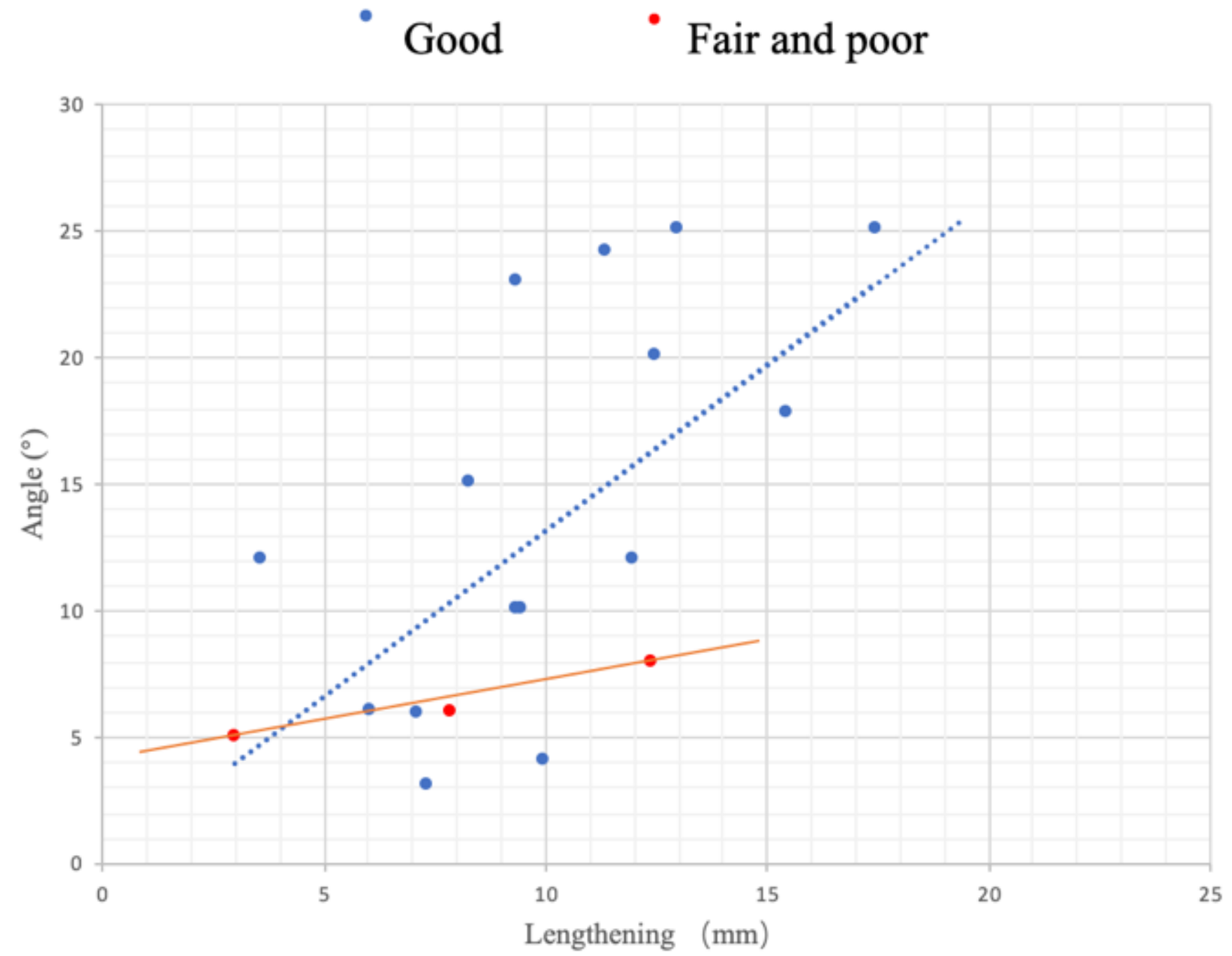

Figure 3

Scatterplot showing the relationships between the lengthening, angulation of ulnar, and the final reduction status. The lengthening was obviously proportional to the magnitude of angulation of ulnar in good outcome patients $(r=0.637, p=0.009)$, and the index was larger than the failed group. 


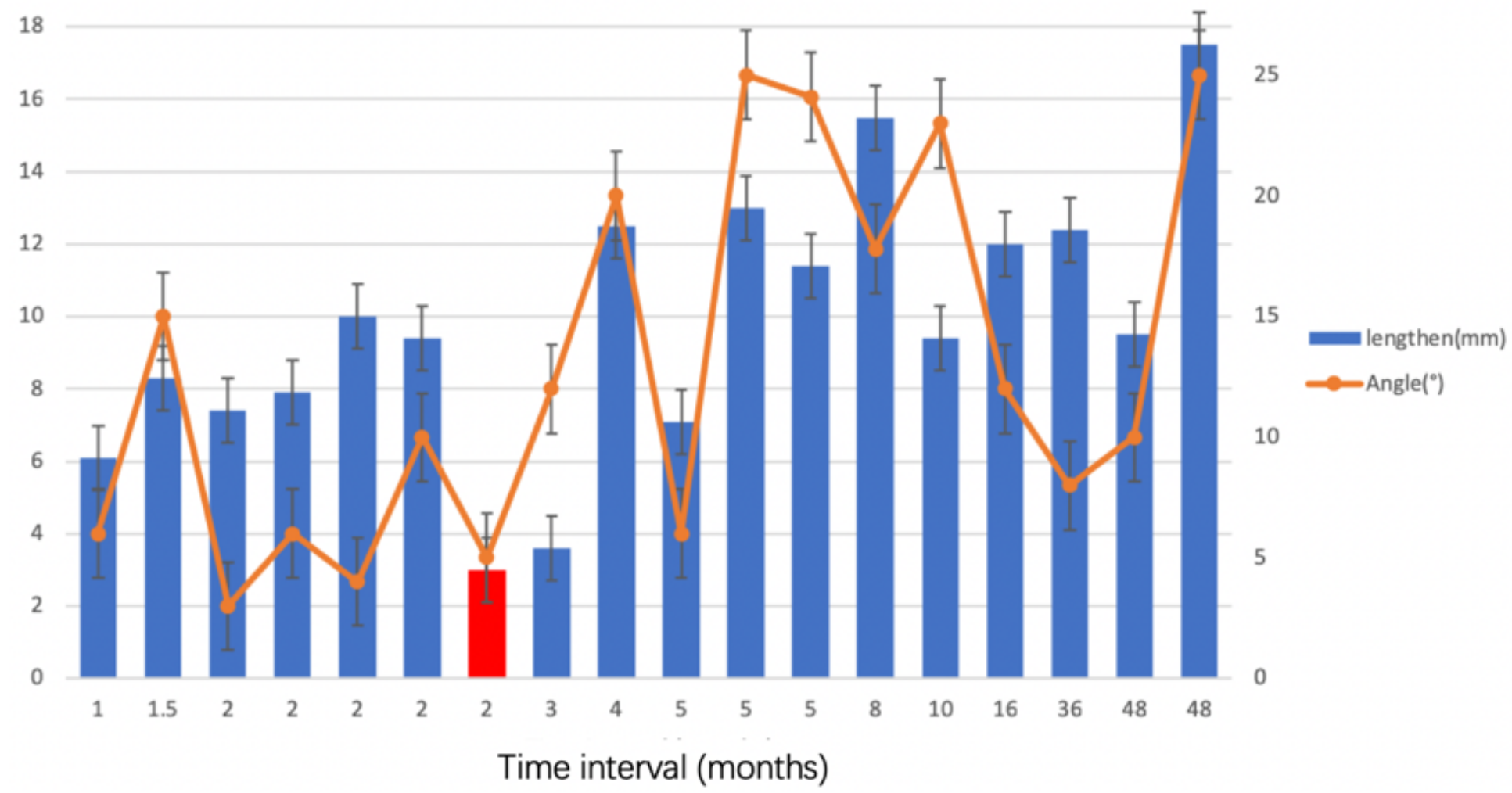

Figure 4

Histogram and Scatterplot shows the relationships between the lengthening, angulation of ulnar, and the time interval. No apparent correspondent relationship was noticed. 